\title{
The relationship between vitamin $D$ supplementation and mortality in critically ill patients
}

\author{
HK Atalan ${ }^{1 *}$, B Gucyetmez ${ }^{2}$, T Sarikayo ${ }^{3}$, UA Turan ${ }^{4}$, E Ozden 5 , M Berktas ${ }^{6}$, N Cakar ${ }^{7}$ \\ From ESICM LIVES 2015 \\ Berlin, Germany. 3-7 October 2015
}

\section{Introduction}

Vitamin D is a fat soluble vitamin that play a major role in the regulation of bone metabolism, and has effects on immun, cardiac and vascular systems [1]. Vitamin D deficiency is common in the general population as well as the critically ill patients and was reported to be associated with increased mortality and morbidity $[2,3]$.

\section{Objectives}

To evaluate relationship between vitamin D supplementation at the ICU admission and mortality.

\section{Methods}

Upon the approval of local ethical committee, a total of 491 patients admitted to ICU of two centers between January 2014 and January 2015 were evaluated retrospectively. The patients who were under 18 years old, elective surgery and whose serum vitamin D levels and outcomes were unknown were excluded from the study. A total of 213 patients were included in the study and divided into 2 groups in accordance with their vitamin D serum level as low Vit-D group $(<25 \mathrm{ng} / \mathrm{dL})$ and normal Vit-D group $(\geq 25 \mathrm{ng} / \mathrm{dL})$. Patients with low serum vitamin D level received vitamin $\mathrm{D}$ orally or via nasogastric tube once at a dose of $600000 \mathrm{iu}$. The patient's age, gender, APACHE II scores, number of failed organ systems, serum vitamin $D$ levels, length of ICU stay and mortality were recorded. Groups were compared by using Mann Whitney U test due to non-normal distribution pattern.

\section{Results}

Low Vit-D group was made up of $166(78 \%)$ patients and normal Vit-D group was made up of $47(22 \%)$ patients. Groups were similar in terms of age (61 vs 63), gender (male, $65 \%$ vs $68 \%$ ), APACHE II score (21 vs 20 ), number failed organ systems (1 vs 1 ), length of ICU stay (12 vs 10) and the ICU mortality ( $22.9 \%$ vs $17 \%$ ) ( $p>0.05$ for each).

\section{Conclusions}

Vitamin D deficiency was found to be frequent at the ICU admission in our study. Mortality rates were similar in both groups, therefore vitamin D supplementation may have beneficial effects on mortality in critically ill patients with low serum vitamin D levels in ICU admission.

\section{Authors' details \\ ${ }^{1}$ Atasehir Memorial Hospital, Anesthesiology and Reanimation, Istanbul, Turkey. ${ }^{2}$ Acibadem International Hospital, İntensive Care, İstanbul, Turkey. ${ }^{3}$ Acibadem Kozyatagi Hospital, İntensive Care, İstanbul, Turkey. ${ }^{4}$ Acibadem Kadikoy Hospital, İntensive Care, İstanbul, Turkey. ${ }^{5}$ Antalya Memorial Hospital, İntensive Care, Antalya, Turkey. ${ }^{6}$ PEPIRC, Yeditepe University, İstanbul, Turkey. ${ }^{7}$ Acibadem University Medical Faculty, Anesthesiology and İntensive Care, İstanbul, Turkey.}

\section{Published: 1 October 2015}

\section{References}

1. Hewison M, et al: Mol Cell Endocrinol 2004, 215:31-38.

2. de Haan K, et al: Crit Care 2014, 18:660.

3. Jeng $L$, et al: J Transl Med 2009, 7:28. 